\title{
Le conflit entre innovation technologique et changement culturel
}

\author{
Manuel MARTIN SERRANO
}

Incluye también la reseña de este artículo publicada en el monográfico de la revista Anthropos dedicado a la obra de Manuel Martín Serrano (no 41-42, 1984, pp. 27-28).

\section{REFERENCIA PARA LAS CITAS DE ESTA PUBLICACIÓN Y DE SUS CONTENIDOS:}

MARTIN SERRANO, Manuel (1976): "Le conflit entre innovation technologique et changement culturel", Internationale Zeitschrift für Kommunikationsforschung, no 1, pp. 25-39. Colonia.

Recuperado el_de de 2 de http://eprints.ucm.es/11052/

\section{UTILIZACIÓN DE ESTE DEPÓSITO:}

Usted es libre de copiar, distribuir y comunicar públicamente la obra bajo las siguientes condiciones, que corresponden a la licencia Creative Commons que protege este texto:

Reconocimiento. Debe reconocer y citar al autor original, utilizando la "REFERENCIA PARA LAS CITAS DE ESTA PUBLICACION Y DE SUS CONTENIDOS" (véase recuadro superior).

No comercial. No puede utilizar esta obra para fines comerciales.

Sin obras derivadas. No se puede alterar, transformar, o generar una obra derivada a partir de esta obra. 


\title{
VINCULACIÓN DE ESTE DEPÓSITO CON OTROS TEXTOS DE MANUEL MARTÍN SERRANO REFERIDOS A “INSTITUCIONES MEDIADORAS Y CAMBIOS SOCIALES”
}

\author{
Presentación y estudio documental por Daniel Franco Romo
}

En E-Prints se tiene acceso a una selección de la obra original de Manuel Martín Serrano (véase: "Publicaciones de Manuel Martín Serrano disponibles en E-Prints. Selección sistematizada"*, en http://eprints.ucm.es/11107/).

Una parte importante de dicha obra está referida a las mediaciones sociales, en sus dimensiones teóricas, históricas, metodológicas y aplicadas. Los desarrollos que se refieren a INSTITUCIONES MEDIADORAS Y CAMBIOS SOCIALES están representados en E-Prints por las siguientes publicaciones:

- "Mediar es operar con la acción que transforma, la información que conforma, y la organización social que vincula, para introducir un designio" (http://eprints.ucm.es/11051/).

- Este documento: "Le conflit entre innovation technologique et changement culturel". Incluye también la reseña de este texto publicada en el monográfico de la revista Anthropos dedicado a Manuel Martín Serrano.

- “«La globalización» es un gigantesco mecanismo de mediación social” (http://eprints.ucm.es/11054/).

\section{REFERENCIAS para enlazar este documento con los que cita y con aquellos que le citan}

- “Prólogo para La mediación social en la era de la globalización” (http://eprints.ucm.es/10651/).

- "Mediación" (http://eprints.ucm.es/10657/).

*Esta selección y sistematización de publicaciones de Manuel Martín Serrano se basa en los análisis realizados por los especialistas que han participado en dos monográficos dedicados a la obra del autor: el primero editado por Anthropos y preparado por Esteban Mate y el segundo por Chasqui, coordinado por Francisco Bernete. Manuel Martín Serrano ha supervisado los trabajos. 


\section{Le conflit entre innovation technologique et changement culturel}

\section{Le conflit de base suscité par le milieu artificiel}

La présente étude entre dans le cadre général des relations entre innovation et changement culturel. L'innovation se réfère à l'apparition d'instruments et de méthodes qui transforment les technologies. Le changement culturel se réfère à la modification des valeurs et des attitudes.

Il existe un niveau d'analyse où l'on pourra distinguer la technologie et la culture, en tant que structures, et l'innovation et le changement, en tant que processus:

$\begin{array}{lll}\text { Niveau de la } & \text { Technologie } & \text { Culture } \\ \text { structure: } & & \\ \text { Niveau du } & & \\ \text { processus: } & \text { Innovation } & \text { Changement }\end{array}$

\section{L'homme dans le milieu artificiel}

En fait, depuis le XIVème siècle on note une tendance à réunir dans la même catégorie la Technologie et la Culture, pour les opposer à la Nature:

Nature $/ / \quad \begin{gathered}\text { Technologie } \\ \text { Evolution } / / \\ \begin{array}{c}\text { Changement } \\ \text { culturel }\end{array}\end{gathered}$

L'humanisme occidental a confié à la nature la fonction de fond, et aux œuvres de l'homme la fonction de figure. Une distinction s'est établie entre les choses engendrées dans la nature et les objets fabriqués: les choses ne sont soumises qu'aux lois du milieu naturel, les objets sont soumis en outre aux exigences du milieu intentionnel crée par l'homme ${ }^{1}$. La pensée humaniste met l'accent sur l'opposition entre deux types de milieux, et deux 
types de besoins: d'une part le milieu naturel, d'autre part le milieu artificiel. Les processus du premier sont régis par des lois, les processus du second par des contraintes ${ }^{2}$ :

(choses)

Milieu naturel

Loi (objets)

Milieu artificiel

Contrainte

Cette façon de situer l'homme et ses œuvres dans le monde n'était pas généralisée avant l'humanisme, et ne s'est pas manifestée dans toutes les cultures. C'est pourtant un trait caractéristique de la nôtre. Nous devons en effet imaginer le monde avant la Renaissance à travers d'autres catégories. L'homme médieval faisait la différence entre technologie et culture, mais il considérait la première comme un reflet de la nature, et la seconde comme un reflet du surnaturel. Au Moyen-Age, il y eut des innovations appréciables. Pourtant elles n'ont pas approfondi le fossé entre milieu naturel et milieu artificiel. Les technologies étaient primitives, non parce qu'elles étaient «rustiques», mais parce qu'elles s'éloignaient très peu des processus naturels. Les outils médiévaux sont en bois et en fer; il faut les comprendre non comme des prototypes grossiers de nos machines chromées, mais comme des répliques très évoluées d'une main, d'une denture, d'un bec ou d'une griffe. Les procédés technologiques imitaient les forces du milieu naturel. Les techniques qui perçaient, frappaient, grattaient, traînaient, ou tournaient, étaient des manières assez précises de multiplier les processus naturels, tels qu'ils sont perçus à l'échelle humaine, plus concrètement à l'échelle de l'œil'3.

On qualifiait d'artificiels ceux des effets naturels qui échappent à la perception, et parmi eux une grande partie des phénomènes biologiques et chimiques. Chimie et médecine étaient suspectes. Elles n'ont pas vu reconnaître leur place parmi les »métiers «, parce que leurs procédés technologiques, par exemple la transmutation chimique, étaient considérés à l'époque médiévale comme contre-nature, »obscurantistes«. Elles ont dû évoluer vers l'alchimie et la pratique des guérisseurs. L'orientation de l'innovation au Moyen-Age explique en partie les caractéristiques »naturelles « d'un milieu humain, et d'un temps humain, très différents des nôtres. Si on explique ces différences par l'idée de progrès, qui est totalement dépourvue de pertinence au Moyen-Age, on effectue une réduction simpliste. Le Moyen-Age utilisait ce type de pensée qui aurait pu servir à développer une technologie fondée sur des processus logiques. L'aptitude à réduire la réalité à des modèles n'a jamais été aussi développée. Si l' "Ars Combinatoria " de Raymond Lulle n'a pas servi à fabriquer un environnement artificiel, c'est parce que seul l'espace naturel était considéré comme humain. 
L'œuvre de l'homme et l'œuvre de la nature étaient des transformations l'une de l'autre. L'arbre, travaillé par l'homme, céderait sa matière au meuble; le meuble, travaillé par le temps, se fendrait comme un arbre, et rendrait finalement sa matière à la nature. Les relations entre le milieu humain et le milieu naturel étaient cycliques: le produit humain était une métaphore de la chose naturelle et la chose naturelle une métaphore de l'objet humain. Ce type de représentation est totalement distinct de celui qui est suggéré par un milieu artificiel. Nos meubles en polyvinyle sont les signes des concepts. Ils expriment une qualité du milieu artificiel: peut-être le confort ou la fonctionnalité. Ils ne retourneront jamais au stade d'éléments naturels. Nos dépôts d'ordures accumulent des fonctions momifiées, des signes qui refusent de disparaître. Ce concept même: »dépôt d'ordures«, espace qui prend, vole le milieu naturel sans le féconder, n'était pas significatif au Moyen-Age. L'évidence du caractère artificiel de nos objets technologiques nous empêche de voir que pour les médiévaux le caractère naturel des leurs était tout aussi évident.

\section{Le mythe du retour à la nature}

L'humanisme a structuré les relations entre milieu naturel et milieu humain, en les définissant par leur mutuelle opposition. Cette pensée a donné naissance à l'un des thèmes les plus caractéristiques de la Modernité, dans les pays de culture latine ou germanique: le conflit entre la vie conforme aux lois naturelles et la vie conforme aux contraintes établies par l'homme lui-même: "La position de l'homme de la Renaissance face à Dieu et à l'univers infini est analogue à celle du Ganymède de Goethe: l'homme est un contenant contenu... La philosophie de la Renaissance, même si elle ne parvient pas à surmonter l'antinomie dialectique qui renferme cette double relation, a le mérite d'avoir signalé le problème et de l'avoir introduit dans une nouvelle conception« (Cassirer, 1951, p.237).

Notre culture tire à la fois ses satisfactions et ses insatisfactions de l'antithèse entre société et nature. Ce n'est pas une contradiction découverte par la sociologie, dans le monde actuel soumis à la rigidité des contraintes et à la lassitude de la surabondance; mais une crise structurelle que a débuté et a été formulée, il y a quatre cents ans.

A partir de la Renaissance, la Nature devient le Paradis Perdu. La Nature est comprise comme une anti-société: fond sur lequel se dessine la figure d'un homme qui fabrique son propre milieu et se crée lui-même: l'homme renaissant qui va engendrer l'homme bourgeois. Ce paradis ne peut être regretté que par une société dont l'enfer est celui peint par Jérôme Bosch: un milieu artificiel peuplé d'objets qui sortent de la tête, qui prennent possession du corps humain et qui soumettent nos mouvements 
et nos actes à leurs propres rythmes et à leurs propres finalités. La nature ne représentera pas le Paradis pour d'autres cultures dont le milieu ne serait pas artificiel.

Avec l'humanisme apparaît une nouvelle dignité pour l'homme: Le concept de liberté conçue comme l'émancipation du joug des besoins naturels, et l'imposition au monde du dessein humain; nait aussi l'angoisse caractéristique du retour à la nature perdue. Une constante culturelle depuis Milton jusqu'aux hippies, qui fournit leurs thèmes à la littérature, aux mouvements sociaux, à la sociologie elle-même: la composante faustienne de notre culture ${ }^{4}$. Il en resulte que le renoncement à l'artificialité du milieu humain et à l'angoisse qui en découle, équivaut au renoncement à l'humanisme. Le retour à l'état de nature représente une forme d'évasion qui (pour employer les termes de Marx), poussée à ses dernières conséquences, instaurerait le faux bonheur de la vie simple des pauvres. Cette évasion est une régression.

Depuis Hegel, nous savons qu'il n'y a pas de liberté pour un sujet, sans qu'il y ait d'aliénation pour un objet. Avec le terme aliénation, il est possible de se référer aussi bien à une condition inhumaine qui se définit comme une transformation des relations entre les hommes, en des relations entre les choses - qu'à la plus humanisée des conditions, qui veut que l'homme s'exprime à travers ses productions: il introduit des relations entre les choses, pour leur donner une finalité spécifiquement humaine. On ne peut mettre un terme à toute forme d'aliénation, sans renoncer en même temps à l'humanisation du monde, et au concept de sujet lui-même. En réalité, la réalisation de la liberté de l'homme en tant qu'opposée au besoin de la nature, exige la réalisation d'une aliénation qui transforme les relations entre les choses, en accord avec les buts de l'homme qui les utilise comme des objets. De ce point de vue, la tentative d'échapper à nos objets techniques a un caractère mythique: logiquement cohérente, elle est objectivement incohérente. L'aliénation humaine provient de ce que l'homme est soumis aux mêmes lois que les choses: les choses sont désaliénées, et l'homme chosifié. L'émancipation humaine suppose par définition l'aliénation des objets à l'homme: l'homme est désaliéné, et les choses, humanisées, transformées en objet pour un sujet. Cette contradiction entre l'état des choses et l'état de l'homme signifie qu'on ne peut pas vouloir en même temps un homme libre et une nature qui ne lui soit pas soumise: en d'autres termes une nature livrée à sa propre spontanéité et un homme non aliéné. La contradiction entre nature et humanisation ne peut être éliminée qu'au niveau »logique «, c'est-à-dire comme un mythe: le mythe de l'homme-nature ou de la nature anthropomorphe. Si l'homme vit d'une manière déshumanisée, c'est parce que les choses sont encore libres, et non l'inverse: l'industrialisation envenime le milieu parce que la 
production n'a pas soumis les choses à des fins humaines: là où l'homme n'est pas maître de la matière, le déterminisme naturel réapparaît. La pollution est la conséquence de la matière abandonnée par l'homme à sa propre spontanéité.

L'idéologie naît moins de l'état d'aliénation que du fait que nous nous supposons inaliénables, c'est-à-dire protégés des relations qui nous transforment en choses, ou sinaliénateurs «, c'est-à-dire capables de réaliser nos desseins de protagonistes du monde, sans le transformer en un objet contrôlé artificiellement. Le mythe du cybernanthrope (ct. Lefebvre, 1971) existe sans aucun doute: celui qui supposerait possible un homme non aliéné dans une société parfaitement programmée. Mais il y a également le mythe du bianthrope: celui qui imagine un homme libre dans une nature spontanée, citoyen d'une Arcadie où seraient possible en même temps la nature non aliénée et la société humanisée. »Après tout, peut être n'est-il pas moins nécessaire de nous comprendre et de nous resituer dans une nature que nous avons faite que dans une nature qui nous a faits « $(S$. Moscovici, 1972, p. 108).

Dans une situation de conflit, les êtres vivants doivent confier la solution à la nature. En dernier ressort, c'est le hasard qui trouve l'équilibre. En dehors du hasard, il n'existe qu'une seule institution capable de médiation: la société humaine. Cette capacité est plus caractéristique de l'homme que l'habilité à se servir d'instruments et à se spécialiser dans les tâches: il semble que ces deux facultés se développent dans les sociétés animales, avec le seul recours de l'ajustement biologique, alors que les ajustements de la médiation sociale nous conduiraient à trouver au fond des processus un développement non des lois naturelles, mais contre les lois naturelles. La société qui médiatise entre les besoins et les buts, substitue les contraintes aux lois. C'est l'organisation sociale, et non la sélection naturelle qui détermine la survivance dans la société humaine. L'ajustement n'est pas dicté par les exigences de la biologie ou de la nature, mais par celles d'un milieu artificiel qui est le milieu naturel pour l'homme socialisé. La société n'est pas et ne pourra pas être un phénomène naturel. On peut imaginer un état de nature dans lequel les hommes seraient soumis exclusivement aux exigences de la sélection naturelle. Ce qu'on ne peut pas imaginer, parce que c'est une contradiction de principe, c'est que dans une telle situation il puisse exister un choix. Le »retour à la nature est une aspiration qui introduit un point de vue correcteur de l'éloignement de la nature, mais personne ne peut vouloir fonder sur la nature aucun humanisme. La communauté hippie isolée qui récolte les fruits de la terre peut survivre, parce que non loin d'elle se trouve un milieu artificiel bien développé. Nous ne faisons même pas allusion aux biens et aux services concrets par lesquels la société environnante soutient une 
communauté qui s'est retirée dans le désert: nous parlons surtout de l'idéologie de ceux qui ont choisi l'éloignement. Ils recherchent un amour non aliéné par les relations fonctionnalisées, mais pas non plus par la nature qui élimine les défavorisés. Ils préfèrent l'activité ludique au travail sans signification. Mais leur but est la jouissance et non l'apprentissage comme chez les animaux. De plus ils veulent une activité ludique qui ne soit pas limitée à l'enfance, quand la mère prenait en charge la lutte pour la vie. L'acceptation des faibles, et la jouissance qui transcende la biologie, supposent des valeurs crées par la vie sociale, et non les lois de la nature. Valeurs qui supposent, à leur tour, les excédents de biens et de temps, conquis dans la lutte contre le milieu naturel. Les programmes idéologiques d'évasion vers la nature ne sont pas naturels. La nature n'obéit à aucun dessein intentionnel: elle ne produit aucune conception «naturelle» de la société: même la société n'est pas son produit, mais le produit d'un détournement contre nature obéissant à l'intentionalité humaine. Si on veut se réclamer d'un programme naturel dans la nature, on ne trouvera que des processus orientés vers l'entropie, en conflit avec le milieu humain artificiellement organisé. Tout programme incluant des valeurs et des intentions supposées naturelles, introduit un ordre qui est celui de l'homme.

Ces mythes qui nous invitent à nous évader d'un monde humanisé vers une vie naturelle, irréelle et irréalisable, ou à ignorer notre nature biologique d'êtres naturels, pour nous perdre dans un monde formalisé non moins irréel et non moins irréalisable, sont spécifiques de notre culture. Si l'époque moderne a séparé milieu naturel et milieu humain, la tentative de les réunir mythiquement apparaîtra sous plusieurs formes successives, tant que notre société pourra être comprise selon le modèle humaniste du monde. La volonté de supprimer les mythes serait vouée à l'échec, si l'on admet que leur fonction est de rétablir au niveau de la représentation du monde une image cohérente de la réalité dissociée. Le mythe nous explique le type de dissociations caractéristiques d'une culture, et dans ce sens, il est un instrument de connaissance. Mais si tout mythe objetive une dissociation réelle de la culture, il propose par définition les solutions irréelles aux conflits culturels: aucun mythe ne peut servir de modèle valable pour l'action social. Utilisé sous cette seconde forme que nous avons caractérisée comme évasive, le mythe agit comme un placebo: il retarde la compréhension des options possibles qui s'offrent à l'homme.

\section{L'humanisation du milieu artificiel}

La voie ouverte pour affronter les angoisses de la société surgie de l'humanisme passe par Galilée, par Marx, par Freud, par Wiener; auteurs qui ont cru à la possibilité d'étendre la liberté humaine, dans la mesure où 
la nature serait aliénée par l'homme, sans perdre de vue que l'homme lui-même est un organisme naturel. Ils nous ont montré que l'évasion ne nous rend pas plus heureux, et que la formalisation de la réalité ne nous rend pas plus humains; que «L'homme qui n'avait pas de chemise» n'était pas heureux, et que Faust n'était pas libre; que le bonheur ne naît pas de la spontanéité de la nature, mais de la capacité d'en finir avec les besoins insatisfaits; et que l'humanisation de la détermination ne se confond pas avec l'absence de détermination, mais avec la capacité de choisir entre des alternatives pouvant être mises en pratique et donnant libre cours à la créativité.

Nous préférons la fleur que nous offre le hippie au formulaire que nous impose le technocrate. Mais l'histoire montre que l'évasion du monde réel, par la fleur, crée des martyrs de la même façon que l'évasion par le formulaire crée des bourreaux. II nous semble aussi nécessaire de rendre impossibles les seconds que les premiers. Face à l'évasion de celui qui fuit le milieu humanisé, et l'évasion de celui qui le déshumanise, les auteurs que nous avons cités ont cru que nous devions disputer le milieu humain tant à la nature qui le dégraderait au point de la désorganiser qu'aux codes qui l'articuleraient au point d'empêcher tout mouvement. Nous avons montré que le thème du conflit entre l'homme et le milieu crée par lui est un trait différentiel de notre culture depuis les origines de l'époque moderne. On ne peut pas considérer qu'il a été découvert par la sociologie critique dans sa méditation sur la société bureaucratique et de gaspillage; il précède aussi la méditation existentialiste sur l'absurde de l'être jeté dans le monde; et il était déjà présent avant la formulation de l'aliénation par Feuerbach et les jeunes hégéliens ${ }^{5}$. Nous nous sommes efforcés de montrer que s'il était possible d'extirper ce qu'il y a d'anti-naturel dans le milieu humain, ce serait alors la fin d l'idée même de l'homme qui nous a été transmise par l'humanisme. Et enfin nous suggérons que l'étude sociologique du milieu artificiel peut et doit montrer ce qu'il a d'insupportable, non parce qu'il serait anti-naturel, mais parce qu'il est encore inhumain.

\section{Modèles théoriques du milieu artificiel}

Du milieu artificiel nous viennent la liberté et la coercition, la satiété et l'insatisfaction, en tant que situations humainement vécues. C'est donc dans le milieu artificiel que nous devons gagner ou perdre la partie contre la pénurie et contre la loi du plus fort. Le résultat dépendra certainement de nos ressources culturelles, et de la direction adoptée par le changement culturel; mais aussi de nos technologies et de l'orientation donnée à l'innovation ${ }^{6}$. Culture et technologie ont en commun d'être des produits humains qui construisent le milieu artificiel. A partir de cette observation, ce qui nous intéresse, c'est ce qui les différencie, puisque nous avons déjà 
insisté sur ce qui les unit. Rappelons encore les éléments que nous envisageons:

Niveau de structure : Technologie

Niveau de processus : (innovation)
Culture

(changement culturel)

Quelles relations y a-t-il entre ces structures et leurs processus correspondants? Sont-ils interdépendants, indépendants ou bien l'un des extrêmes est-il à l'origine de l'autre? Examinons les alternatives possibles.

\section{Modèles de type inductif}

Ceux-ci introduisent une relation entre structures et processus. Ils soutiennent l'hypothèse que l'innovation technologique provoque le changement culturel, ou viceversa. Quand on considère que le moteur du changement se trouve dans la technologie, et que les changements culturels sont des effets des transformations qui s'opèrent dans les techniques, nous nous trouvons devant la vision technocratique de la culture. Ce point de vue, dans sa version extrême coïncide avec une vision matérialiste de la culture: le célèbre slogan de $M c L u h a n$ en est un exemple: "le message est le medium». D'autres interprétations plus limitées reconnaissent que la culture est relativement autonome: mais elles insistent sur le fait que la plus grande part du changement est induite par l'innovation. Le changement culturel s'entend, fondamentalement, comme l'acquisition de nouveaux savoir-faire permettant d'utiliser les instruments et procédés techniques. En dernière instance, l'innovation technologique est le moyen et en même temps l'essentiel du contenu culturel. Quand on parle de planification éducative, on se réfère généralement à cette dernière forme du modèle technocratique.

Une autre hypothèse orientée en sens inverse, suppose que le changement culturel est le moteur des changements technologiques: chaque culture s'appliquerait à développer la technologie exigée par son évolution. On souligne le fait que le changement est immanent à la culture, et l'innovation sa conséquence. La culture est interprétée comme la créativité elle-même. Le changement culturel cesse quand la créativité s'épuise, bien que la technologie continue à développer les innovations induites par les étapes culturellement créatives. Spengler est un représentant de cette hypothèse. La même idée sur l'origine de l'innovation dans la créativité culturelle se trouve dans le surréalisme. La tentative pratique de confectionner des objets technologiques répondant au changement culturel s'observe dans le développement du projet industriel, ou du prototype fonctionnel. 
Ceux-ci introduisent des relations à double sens entre les structures et aussi entre les processus. Tout changement social produirait une innovation, et toute innovation technologique produirait une réponse culturelle. $\mathrm{Ce}$ processus étant cyclique, il sera inutile de s'interroger sur son origine: nous remonterions au premier outil technologique et au premier signe culturel pour nous trouver devant un problème de priorités aussi vain que celui de l'œuf et de la poule. Ce modèle élimine la nécessité de choisir entre l'ajustement du changement culturel aux processus technologiques, et l'ajustement des innovations technologiques au changement culturel, mais seulement à condition de prendre en considération un autre niveau de décision, distinct à la fois de celui de la technologie et de la culture. C'est pourquoi on tend à voir l'innovation et le changement comme des processus solidaires, et on les inclut à l'intérieur d'une seule et même catégorie, que nous pourrions appeler fonctionnelle. Les transformations de la technologie et de la culture seraient solidaires par rapport à toute autre instance et l'attention devra se concentrer sur le niveau par rapport auquel se produit l'ajustement.

La théorie de changement fonctionnaliste, mais aussi la planification socialiste, entreraient dans cette catégorie. La différence provient de l'importance plus ou moins grande accordée à l'un ou l'autre élément du cycle; la ressemblence se trouve dans la soumission à un système extérieur qui laisse neccessairement dans l'ombre les tensions entre innovation et changement. L'induction est donc transposée à l'extérieur du système culturel-technologique. Pour les fonctionnalistes, le système a toujours fini par laisser le dernier mot à la loi naturelle de l'évolution, c'est-à-dire à la nature; c'est ainsi qu'ils se sont éloignés du niveau d'analyse sociologique. Cela s'était déjà produit dans la sociologie de Spencer, et vient de se reproduire par l'œuvre de T. Parsons: on peut s'en assurer en lisant: «Societies: Evolutionary and Comparative Perspectives» (Englewood Cliffs 1966). Pour les socialistes, le dernier mot est laissé au parti, interprété comme l'objectivité même. On introduit ainsi un volontarisme formaliste, que l'on peut vérifier dans l'œuvre de Lukács, par exemple: «Raison et révolution» et «Esthétique».

\section{Modèles de la médiation}

Un modèle de la médiation introduira, à l'intérieur de chaque niveau et entre chaque niveau et à la place des flèches indiquant une dépendence, des barres qui indiquent des structures et des processus directement irréductibles. Innovation et changement culturel sont deux systèmes irréductibles l'un à l'autre, parce que les phénomènes de chacun d'eux 
obéissent à des déterminismes différents. L'innovation intervient nécessairement dans le cadre des restrictions technologiques, le changement culturel nécessairement dans le cadre des restrictions culturelles. Pourtant, leurs effets s'appliquent à l'intérieur d'un seule et même objet: la société.

L'accord social des deux processus voit son action limitée par les degrés respectifs de liberté que peuvent adopter l'innovation et le changement culturel. La capacité de la société de maîtriser leurs effets se réduit au fur et à mesure que la complexité du milieu augmente: il n'y a donc rien d'étonnant à ce que les options sociales soient plus limitées là où la technologie est la plus innovatrice et la culture la plus qualitative. Le désajustement qui affecte la société comme conséquence de la rencontre entre les technologies et la culture, provient de la nature hétérogène des deux processus. Les désajustements pourront sans doute être moins accusés, mais en aucun cas éliminés.

\section{La médiation en tant que catégorie du changement social}

La société se voit soumise à la pression de contraintes dues à l'innovation et incompatibles avec les contraintes imposées par la culture: c'est-à-dire qu'elle se trouve dans une situation dissonante. Une grande part des énergies sociales vise à reduire la dissonance, à établir le meilleur ajustement possible entre innovation et changement. Ce sont précisément les énergies consommées dans la médiation. Bien que le processus médiateur consomme lui-même peu d'énergie, les investissements en capital matériel et humain nécessaires pour l'assurer sont cependant importants. C'est notamment le cas de l'éducation permanente, et des normes de récyclage, de la pschothérapie, des institutions religieuses, des entreprises officielles qui s'occupent de l'assistance sociale. En tant que producteurs de modèles d'ajustement pour réduire la dissonance, ils méritent d'être distingués des autres secteurs de l'activité sociale.

Il est évident qu'on ne peut pas imposer au déterminisme technologique les lois du déterminisme culturel. Il semble difficile que l'innovation introduite par les contraceptifs puisse être maîtrisée par des normes sexuelles imposées à la femme dans les sociétés patriarcales. Il est tout aussi peu probable que les changements culturels liés à cette innovation se soumettent au déterminisme de la technologie anticonceptionnelle: les contraintes culturelles éviteront la disparition de la fécondation, bien que, techniquement, elle puisse être éliminée. L'usage que la femme fera de son émancipation sexuelle dépendra davantage du degré de liberté toléré par le changement des normes sexuelles, que de la tolérence organique des contraceptifs. La dissonance entre une technologie antifécondatoire et des normes fécondatoires évite que la société se réorganise spontanément, avec le seul but d'exploiter au maximum les opportunités techniques 
permises par l'innovation. Elle empêche également que la société se fossilise spontanément dans ses normes culturelles, pour préserver on ne sait quel type de valeurs imperméables au changement.

Cet exemple montre bien qu'une innovation surgie du laboratoire a davantage accéléré le changement des normes concernant la femme que de longues années de lutte pour l'émancipation, au niveau des principes. Des valeurs et des attitudes historiquement aussi «essentielles», comme celles qui se réfèrent à la vertu et à la procréation doivent changer leur signification culturelle à cause de l'apparition d'une innovation. Le «contenu» des valeurs n'est donc pas déterminant quant à sa résistance au changement: dans ce cas, il était suffisant - et nécessaire - de faire disparaître une contrainte physiologique qui imposait des limites à la femme plus efficacement que le droit patriarcal. En même temps, cet exemple montre que l'innovation apparue avec les contraceptifs n'agira qu'à son niveau spécifique, en accord avec ses vertus techniques: le contraceptif est un inhibiteur de processus chimiques et non de valeurs culturelles. Notamment, l'utilisation des contraceptifs est rapporté aux normes différentes dans les pays sous-développés («normes de subsistance») et dans les pays sur-développés («normes de choix existentielle»). Seuls ceux qui pensent que les normes culturelles sont des produits immédiats des caractères physiologiques, peuvent craindre que les contraceptifs mettent fin à toute différenciation entre les sexes. Le rôle de la femme dans notre société a été techniquement contrôlé par ses caractéristiques physiologiques, plus simplement par les grossesses; mais ce rôle et ce contrôle s'inscrivent dans une organisation sociale caractérisée par le partage inégal de l'héritage culturel et matériel entre les sexes et par la manière de diviser le travail et les privilèges enre eux.

Ces contrôles culturels ont, en même temps que les contrôles physiologiques, une incidence sur les choix de la femme: en la situant dans une position conflictuelle où elle ne pourra ni se soumettre aux exigences culturelles traditionnelles, parce qu'elles sont dissonantes par rapport aux options physiologiques, ni épuiser les ressources qu'offre l'innovation technologique, parce qu'elles sont dissonantes par rapport aux options culturelles. Cette dissonance devra être résolue: ce qui va s'ajuster, ce n'est ni la technologie, ni la culture, mais l'objet sur lequel elies agissent de manière contradictoire, dans ce cas la femme elle-même, en tant que modèle technologique et modèle culturel. On peut trouver dans cette remarque une façon d'exprimer opérationnellement le concept de conflit au niveau de l'être ${ }^{7}$. La forme selon laquelle l'innovation va s'introduire dans la société sera adaptée à la représentation du modèle médiateur; la forme selon laquelle elle va changer les normes culturelles sera adaptée au modèle médiateur. Il n'y a que dans la représentation que l'on peut réduire 
la dissonance entre des processus qui ont une incidence sur le même objet et appartiennent à un plan distinct. Par conséquent, les normes culturelles ne s'adaptent pas à l'innovation technologique sans médiation, et les fonctions technologiques ne s'adaptent pas non plus à la culture sans médiation. Le concept de médiation permet d'échapper au déterminisme mécaniciste, et au fatalisme fonctionnaliste. Le déterminisme mécaniciste croit que le changement s'impose mécaniquement au moyen de l'innovation: c'est la pratique du "matérialisme scientifique», qui depuis la fameuse formule de Lénine est égal à un type d'organisation (les Soviets) plus un type d'innovation (l'électricité). Cette simplification tourne le dos au système théorique de Marx qui a le mieux étudié la médiation. Pour Marx, aucun processus entre la théorie et la pratique n'est concevable sans une médiation protégeant à la fois de l'idéalisme et du mécanisme. Il n'est pas étonnant que l'idée de Lénine de transformer scientifiquement, c'est-àdire mécaniquement, l'homme et sa culture au moyen d'une planification de l'innovation, se soit étendue à des pays capitalistes, sans que ce «matérialisme scientifique» leur ait ouvert la voie vers le communisme. L'hypothèse fonctionnaliste selon laquelle toute innovation qui parvient à s'imposer dans une société est fonctionnelle par rapport à sa culture du simple fait qu'elle existe est fataliste. Elle empêche de voir que la rationalité technologique n'équivaut pas à la rationalité culturelle, et que le principe d'utilité de l'innovation ne permet pas de déduire le principe de cohérence du changement social. Le fait que la société conserve au moins la fonctionnalité nécessaire pour se perpétuer sans grandes secousses structurelles, ne prouve pas que les changements soient à la fois rationnels et cohérents. Ce qui est prouvé c'est qu'une médiation opère entre le changement et l'innovation: elle met en ordre l'incohérence et l'irrationnalité pour les rendre formellement non-contradictoires et matériellement utilisables.

L'hypothèse sociologique selon laquelle la contradiction entre les contraintes technologiques et les contraintes culturelles est le moteur du changement et de l'innovation, nous paraît valable. Mais ce qu'il faudra examiner, c'est son corollaire: que la société ne peut se perpétuer et se reproduire elle-même, en état de contradiction structurelle. Cette conclusion se trouve fréquemment démentie par l'histoire. L'idée que la révolution est la forme que la médiation adoptera nécessairement pour sortir de l'état contradictoire semble se fonder sur un modèle dans lequel ordre et contradiction étaient logiquement opposés. Nous savons aujourd'hui que l'opposition logique s'établit entre ordre et désordre, d'une part, et entre contradiction et simultanéité, d'autre part; qu'il est parfaitement possible, en termes logiques, de construire un modèle d'ordre isomorphe d'une réalité contradictoire, et que l'action sur la réalité, en se servant de ce 
modèle, n'offre aucune difficulté particulière. Ainsi la contradiction peut faire, et fait effectivement partie, de l'ordre; l'irrationnalité structurelle peut être représentée, et perpétuée, au niveau d'un modéle formellement rationnel. L'incohérence dans les relations sociales peut être aménagé et établie, au niveau d'une représentation formellement cohérente. A notre avis, tant que les représentations pourront réduire la dissonance et permettront que les contradictions structurelles puissent être représentées et utilisées comme un type d'ordre - l'ordre contradictoire - l'espoir que la révolution viendra d'elle-même, mécaniquement, n'a pas de fondement historique ni sociologique. La révolution n'a pas surgi nécessairement de la contradiction, parce qu'auparavant la contradiction a cessé d'être la négation de l'ordre par la raison, et un état que l'action planifié ne peut pas imposer et perpétuer.

Le répertoire des représentations, interprété comme l'ensemble des significations partagées, constitue le capital d'une communauté: c'est la matière du consensus auquel est liée la conservation de l'ordre établi. Un objet, un outil qui disparaissent peuvent être substitués, par exemple les minéraux chez les Lapons après leurs migrations. La perte d'une institution culturelle ou politique dont on pensait que dépendait l'existence du système peut être assimilée sans grands bouleversements: par exemple la prise en charge par l'Etat des grands secteurs de la production, dans les pays capitalistes, malgré l'idéologie de la «libre entreprise». Mais la perte d'une représentation rend impossible l'emploi de n'importe quel outil, et l'utilisation de n'importe quelle institution: tel est le cas de l'effondrement des civilisations Maya et Inca à partir du moment où leurs modèles culturels et technologiques sont anéantis par des modèles européens. Nous pouvons imaginer que le retour au néolithique se produirait non par la disparition de toutes les roues, mais par l'oubli du modèle de la transmission mécanique circulaire; que la chute du capitalisme pourrait peut-être se produire quand la représentation de la valeur d'échange pourra être détruite, par la concentration monopoliste, ou l'apparition d'un nouveau modèle d'ajustement entre les efforts productifs et les aspirations.

\section{Anmerkungen}

1 Dans notre analyse nous distinguerons entre chose et objet. La chose se rapporte à la nature, l'objet à la société. La chose devient objet parce que la société s'objective à travers elle. Il faut souligner que la distinction ne s'etablit pas par rapport à l'usage, mais par rapport à la forme de l'utilisation. Autrement dit une chose n'est pas médiatisée, alors qu'un objet est utilisé sous une forme médiatisée. L'objet est le support d'un ordre significatif par rapport à l'homme. Cet ordre est artificiel par rapport à l'ordre naturel. L'homme vit donc dans un milieu constitué de choses et d'objets. Ce milieu est le champ même du conflit entre un système d'ordre réactif, et un système d'ordre projectif. Parce que toute chose peut être 
soumise à des formes d'utilisation spécifiquement artificielles et humaines (par exemple de l'air en boîte). De même chaque objet produit par l'activité humaine est soumis aux lois naturelles. L'évolution de ce conflit n'est rien d'autre que l'évolution des représentations de l'environnement.

2 Nous empruntons le mot «contrainte» à la tradition durkheimienne, en signifiant la coercition spécifiquement produite par la vie sociale en opposition à la coercition naturelle, expression des lois constantes de la nature.

3 Le soupçon que dans la nature il y aurait des processus naturels cachés à la perception immédiate marque la transition vers la philosophie de la nature de la Renaissance. Pic de la Mirandole intègre la magie parmi les sciences, en la considérant comme la connaissance des lois profondes des phénomènes: aujourd'hui nous le considérerions comme un structuraliste (v. Apologia, in Opera, folio 170, Bâle). Joh. Baptista Porta définit le concept de «magia naturalis» (v. oeuvre du même titre, Libri viginti, 1, cap. 2) et l'acceptation définitive d'une science non sensible chez Campanella (v. «Universalis philosophiae» in: «Metaphysicarum rerum juxta propia dogmata partes tres», Paris 1938, P-II, Lib. VI, Cap. 7, et in: «De Sensu rerum et magia», Francfort 1920, Libr. IV, cap. I, pag. 260 seq.). Ce sont les auteurs auxquels nous renvoient Kepler et Galilée.

4 «Son essence est le dépassement de l'apparence sensible; son sentiment, la solitude; son désir ardent l'infini ... Pathos de la distance entre l'homme et la Nature» (O. Spengler, 1966, p. 482).

5 Par exemple chez les puritains, comme le montre Max Weber; dans «l'Ethique» de Spinoza, d'après l'analyse d'Unamuno; dans le «Dialogue sur les deux grands systèmes du monde», de Galilée, quand il analyse les relations entre changement et société.

6 La déclaration des droits de l'homme, le code du mariage, sont certes des objets culturels importants; mais les mass media et les produits surgelés sont des objets technologiques non moins déterminants.

7 A partir de la Renaissance notre culture évite l'application d'un ajustement qui subordonne la culture à la technologie, ou la technologie à la culture. Elle agit sur le support individuel ou social pour réduire la dissonance. De ce point de vue, la névrose qui exprime le conflit au niveau de l'être apparaîtra comme l'alternative que notre culture a choisi pour fuir la pénurie (ajustement qui subordonne la technologie aux normes) et l'anonymie (ajustement qui subordonne la culture à la technologie).

\section{Zusammenfassung - Summary - Résumé}

Der Aufsatz untersucht die Beziehungen zwischen technologischer Innovation und kulturellem Wandel. Innovation bezieht sich auf das Aufkommen von Instrumenten und Methoden, die die Technologie verändern; kultureller Wandel auf die Veränderung von Einstellungen und Werten. Der Autor untersucht zunächst die Beziehung des Menschen im Mittelalter zu den selbstgeschaffenen Gegenständen. Diese ist noch nicht konfliktgeladen, da die Objekte als Abbild der Natur aufgefaßt werden. Mit der Renaissance beginnt man zwischen natürlichen Dingen der Umwelt und vom Menschen geschaffenen künstlichen Objekten zu unterscheiden. Anschließend untersucht der Autor verschiedene theoretische Modelle der künstlich geschaffenen Umwelt: induktive, Anpassungs- und Vermittlungsmodelle sowie die Vermittlung als Kategorie des sozialen Wandels. Das Konzept der Vermittlung erlaubt uns, sowohl dem mechanistischen Determinismus als auch dem funktionalistischen Fatalismus zu entkom- 
men. Der Gegensatz zwischen technischen und kulturellen Zwängen ist gerade Motor des Wandels und der Innovation.

The essay discusses the relations between technological innovation and cultural change. The term innovation refers to the appearance of instruments and methods changing technology; cultural change is related to the transformation of attitudes and values. First the author studies the relationship of medieval man to objects created by himself. Since the objects are considered as images of nature, this relation does not yet involve any conflicts. The Renaissance begins to differentiate between natural things of the environment and artificial objects created by man. It follows an examination of different theoretical models of the artificially created environment: inductive models, adaptation and mediation models as well as the mediation as category of social change. The concept of mediation permits us to escape not only determinism but also functional fatalism. The opposition of technical and cultural pressures is just the motor (moving force) of change and innovation.

L'auteur examine les relations entre l'innovation technologique et le changement culturel. Innovation se rapporte à l'apparition des instruments et des méthodes qui transforment la technologie; changement culturel se rapporte à la transformation (modification) des attitudes et des valeurs. D'abord, l'auteur explore la relation de l'homme du Moyen-Age aux objets créés par lui-même. Celle-ci ne contient pas encore des conflits, parce que les objets sont considérés comme des images de la nature. Avec la Renaissance on commence à séparer les objets naturels du milieu des objets artificiels créés par l'homme. L'auteur examine des differents modèles théoriques du milieu artificiellement crée: des modèles inductives, d'adaptation et de médiation de même que la médiation comme catégorie du changement social. Le concept de la médiation nous permet d'échapper au déterminisme mécaniciste aussi bien qu'au fatalisme fonctionnaliste. C'est l'antithèse des pressions techniques et des pressions culturelles qui est le moteur du changement et de l'innovation. 


\title{
Reseña de MARTIN SERRANO, Manuel (1976): "Le conflit entre innovation technologique et changement culturel", Internationale Zeitschrift für Kommunikationsforschung, no 1, pp. 25-39. Colonia.
}

\author{
Publicada en Anthropos. Boletín de Información y Documentación, no 41-42, \\ pp. 27-28. Monográfico dedicado a Manuel Martín Serrano.
}

\begin{abstract}
"Este estudio se refiere a las relaciones entre innovación y cambio cultural. "Innovación" se refiere a la aparición de instrumentos y métodos que transforman las tecnologías. "Cambio cultural" hace referencia a la modificación de los valores y las actitudes [...]. Innovación tecnológica y cambio cultural son sistemas irreducibles el uno al otro, porque los fenómenos de cada uno de ellos obedecen a diferentes determinismos [...]. Sin embargo, sus efectos se aplican en el interior de un único y $e$ mismo objeto: la sociedad... Son, precisamente, las energías consumidas en la mediación.»
\end{abstract}

El lector que desee conocer los análisis históricos de los que Manuel Martín Serrano va a concluir con el desarrollo de la "teoría de la mediación", puede encontrar en este artículo, escrito en 1974, algunas claves importantes. Posteriormente, en La mediación social amplía estos análisis y los formaliza; pero en este texto se pone de manifiesto mejor cuál es la lógica que le lleva al autor a dar el paso del estudio de las representaciones colectivas, al análisis de las mediaciones sociales.

El autor muestra los modelos de representación del cambio histórico y social disponibles para explicar las relaciones existentes entre innovación tecnológica y cambio cultural. Señala que no cabe analizar el determinismo tecnológico a partir de las leyes que rigen en los controles culturales, ni viceversa; pero que la sociedad se ve sometida simultáneamente a la presión de ambos tipos de contraintes. Examina con detalle los distintos efectos culturales que tiene en sociedades diferentes, la introducción de una misma innovación tecnológica -por ejemplo, los anticonceptivospara ilustrar la tesis esencial de este artículo: el sistema tecnológico y el sistema normativo no están integrados. La utilidad que "representa" una innovación responde a un principio de racionalidad tecnológica; la coherencia que "representan" unas normas sociales responde a un principio de racionalidad cultural; ambas racionalidades son disonantes en la cultura occidental a partir de la revolución industrial.

Sin embargo, la sociedad capitalista ha conservado la funcionalidad necesaria para perpetuarse; lo cual significa que ha logrado manejar la disonancia entre la presión de las tecnologías y la conservación de las normas. En todos los grupos humanos, una parte de los recursos sociales se destinan a intervenir sobre la conciencia de sus miembros para reproducir una visión del mundo consonante, es decir, una representación de la realidad en que lo que cambia, pueda ser recuperado como componente de la identidad social. Estos recursos, obviamente están al servicio de la reproducción del grupo (económica, institucional, axiológica). Pero a partir del capitalismo in- 
dustrial, y sobre todo en el capitalismo monopolista, la tarea reproductiva de restaurar la consonancia, deriva hacia sí inversiones mucho más importantes que antes en capital material y humano; y sobre todo, se institucionaliza en lo que el autor denomina "instituciones mediadoras": por ejemplo, cumplen esa función las relaciones públicas, el reciclaje, la psicoterapia, los asistentes sociales, la comunicación de masas. Estas instituciones, cuya tarea principal que les asemeja consiste en la producción de modelos mediadores para reducir la disonancia, deben ser estudiadas como un sector reproductivo diferente a los otros sectores de la actividad económica; y la tarea que cumplen -la mediación- es uno de los objetos de estudio estratégicos si se desea entender los mecanismos del control social, del cambio y de la reproducción social.

El autor hace una corrección a la interpretación determinista del marxismo, manteniéndose no obstante fiel a la metodología dialéctica:

"La hipótesis según la cual las contradicciones entre las constricciones tecnológicas y culturales son motores del cambio y de la innovación me parece validas. Pero habrá que revisar [...] que la sociedad no pueda perpetuarse y reproducirse en un estado de contradicción estructural. [...] La idea según la cual, la revolución es la forma que adopta necesariamente la mediación para salir del estado contradictorio, parece estar fundada en un modelo según el cual orden y contradicción se oponen lógicamente. Pero [...] la oposición lógica se establece entre orden y desorden, por una parte; y entre contradicción y simultaneidad, por otra. Es perfectamente posible, en términos lógicos, reproducir el modelo que describe una realidad contradictoria y servirse de ese modelo para actuar sobre la realidad. De esta manera es como la contradicción llega a formar parte del orden: la irracionalidad puede ser representada y perpetuada a nivel de un modelo formalmente racional; la incoherencia en las relaciones sociales puede ser manejada a nivel de una representación formalmente coherente... En mi opinión, y en tanto que las representaciones puedan reducir la disonancia y hagan posible que las contradicciones estructurales pueden ser utilizadas como un tipo de orden -el orden contradictorio- la esperanza de que la revolución llegara por sí misma, no tiene fundamento histórico ni sociológico». 